\title{
Loi Biodiversité et choix de société
}

Natures Sciences Sociétés analyse la mise en politique de la biodiversité depuis la création de la notion ${ }^{1}$. Le projet de loi sur la biodiversité en discussion depuis 2013 et transmise au Sénat en mars $2015^{2}$ nous permet de prendre conscience du chemin parcouru par cette notion, de son ouverture aux gènes, aux paysages, aux milieux marins, etc., et des missions auxquelles elle est associée : capacité d'évolution, services écosystémiques, continuité écologique, solidarité écologique, moteur économique, etc. Elle nous permet aussi, du fait des âpres discussions qui l'entourent, de mesurer les choix de société ainsi que le caractère politique des représentations de la biodiversité encadrées par la loi. On y trouve en effet la plupart des éléments qui rendent compte des relations entre les hommes à propos de leur environnement.

La loi Biodiversité prend donc son temps. Il a fallu 39 ans, après la loi de 1976 relative à la protection de la nature essentiellement dédiée à la protection des espèces, pour que la France rénove sa législation afin de se mettre en accord avec les nouvelles visions scientifiques et sociétales de la biodiversité, de donner une base légale à ses propres stratégies nationales (de la biodiversité, de la transition écologique, de la mer et du littoral...) et aux normes internationales de la convention sur la diversité biologique (1992) et du protocole de Nagoya (2010). Dans sa dernière version transmise au Sénat, la loi arborait le titre guerrier et très ambitieux de « Loi pour la reconquête de la biodiversité, de la nature et des paysages ».

Le terme de reconquête n'a rien d'anodin. L'atteinte des objectifs de biodiversité pose en effet la question de la reconquête de territoires de plus en plus artificialisés $\mathrm{du}$ fait du développement économique. Reconquête signale aussi la volonté de freiner ce mouvement d'artificialisation, ce qui, en France métropolitaine, se traduit

\footnotetext{
1 Voir, par exemple, le dossier consacré à la construction sociale de la question de la biodiversité : Aubertin, C. (Ed.), 1998. Dossier « La biodiversité : un problème d'environnement global ", Natures Sciences Sociétés, 1998, 6, 1 et 2.

2 http://www.senat.fr/dossier-legislatif/pjl14-359.html.
}

par la préservation d'une biodiversité amie de l'homme, façonnée par lui et déjà ancienne, sans forcément anticiper les modifications à venir, en particulier celles liées au changement climatique.

Le projet de loi accuse l'influence des principes de la «nouvelle gestion publique» qui prévalent dans les réformes de l'administration française depuis une vingtaine d'années : le partage des responsabilités avec une compétence décentralisée des régions qui définissent leur propre stratégie, la fusion des administrations et l'optimisation des dépenses publiques. La création de l'Agence française de la biodiversité, regroupant services et budgets, s'inscrit dans cette logique des constantes réductions de moyens. Le contexte économique est également prégnant avec la volonté de soutenir les filières économiques de la biodiversité, "les filières de la croissance verte et bleue dans le domaine de la biodiversité, en particulier le génie écologique et le biomimétisme» $\left(\mathrm{p} .10 \S 10^{3}\right)$. Cette vision économique de la biodiversité se retrouve notamment, parmi d'autres inflexions manifestes, dans le titre IV relatif à l'accès et au partage des avantages (APA) et dans l'article 33 consacré aux procédures de compensations des atteintes portées à la biodiversité.

La question de l'APA, troisième objectif de la convention sur la diversité biologique qui devait associer les pays du Sud aux retombées économiques de l'exploitation des ressources génétiques, est devenue un thème central des négociations et une scène d'affrontement Nord-Sud. La France est particulièrement concernée. Elle utilise et fournit des ressources génétiques, elle possède de nombreuses et anciennes collections d'animaux et de plantes, elle réunit plusieurs territoires d'outre-mer à la grande biodiversité tropicale et marine, enfin, elle compte parmi ses citoyens des "communautés autochtones et locales » non reconnues par la République.

\footnotetext{
3 Toutes les citations dans cet éditorial sont issues du Projet de loi pour la reconquête de la biodiversité, de la nature et des paysages, Doc. parl. S., n 359, 2015, http://www.senat.fr/leg/ pj114-359.pdf.
} 
Malgré les critiques émises depuis plus de vingt ans quant aux faiblesses de la formulation «communautés $\mathrm{d}$ 'habitants » qui ne sécurise pas les populations dans des contextes de changement rapide des modes de vie, le projet de loi entérine ce concept défini comme «toute communauté d'habitants qui tire traditionnellement ses moyens de subsistance du milieu naturel et dont le mode de vie présente un intérêt pour la conservation et l'utilisation durable de la biodiversité » (p. 25 § 20). En cas de connaissances traditionnelles associées, les procédures d'APA « visent à recueillir le consentement préalable en connaissance de cause des communautés d'habitants concernées » (p.31 § 80). Comme dans la récente loi brésilienne sur l'APA, le projet de loi français prévoit que les communautés d'habitants participent aux prises de décision, mais il ne prévoit pas qu'elles décident de l'utilisation de leurs savoirs. Enfin, le retour financier aux populations, pourtant affirmé dans l'esprit de la loi, n'est pas construit légalement et subit de nombreuses restrictions pour « les connaissances traditionnelles associées à des ressources génétiques ne pouvant être attribuées à une ou plusieurs communautés », et pour « les connaissances traditionnelles associées à des ressources génétiques dont les propriétés [...] ont été utilisées de longue date et de façon répétée en dehors des communautés d'habitants qui les partagent » (p. 26 § 39 et p. $27 \S 40$ ). Plus généralement, « lorsque le partage des avantages découlant de l'utilisation des ressources génétiques comporte un avantage financier, celui-ci est affecté à l'Agence française pour la biodiversité » (p. 30 $\S 74)$. Il faudra compter davantage sur la déontologie des chercheurs que sur la loi pour respecter l'équité dans les échanges de substances naturelles avec les populations.

La priorité du titre IV semble être de protéger les collections et herbiers des procédures APA jugées trop contraignantes par les grands organismes de recherche. L'accès à ces collections pourrait être simplifié pour les chercheurs. Enfin, significatif d'une représentation d'une biodiversité "sauvage", le titre IV exclut le domaine agricole et ne considère donc pas les savoirs paysans sur l'agriculture.

Les compensations apparaissent dans le titre $\mathrm{V}$ avec le chapitre II sur les mesures foncières. Le principe de compensation, ultime recours de la doctrine «Éviter, réduire, compenser », et jusque-là essentiellement utilisé dans le cadre des dérogations pour l'atteinte à des espèces protégées à la charge de l'aménageur, prend une nouvelle dimension. Afin de permettre une plus grande efficacité économique et environnementale, « des opérations favorables à la biodiversité, dénommées "réserves d'actifs naturels", peuvent être mises en place par des opérateurs de réserves d'actifs naturels" (p. 55 § 12). L'obligation de compensation pourrait désormais s'effectuer par achat de ces réserves d'actifs naturels auprès d'opérateurs agréés par l'État, comme la Caisse des dépôts et consignations Biodiversité, ou de filiales d'aménageurs, comme EDF, ou même de collectivités territoriales, comme le conseil départemental des Yvelines. Cette " compensation par l'offre », où les responsabilités des acteurs et la pérennité des actifs ne sont pas encore bien définies, laisse entrevoir la création d'un marché de certificats sur le modèle des biobanques américaines et une dérive vers la gouvernance par le marché.

Les questions soulevées par cette loi traversent plusieurs textes qui composent ce numéro: Le dossier «Politiques du changement global. Expertises, enjeux d'échelles et frontières de l'action publique environnementale » expose comment les problèmes globaux d'environnement, comme les transformations accélérées des systèmes socioéconomiques, investissent le champ politique ; le texte d'Audrey Coreau et al. relate l'expertise multiacteurs et multidisciplinaire BioPIQuE qui a permis d'identifier 25 questions émergentes, dont 10 stratégiques, pour les politiques publiques de biodiversité en France métropolitaine d'ici à 2020 ; parmi celles-ci, des interrogations sur la façon de s'appuyer sur la résilience des écosystèmes pour construire les stratégies d'adaptation, la pertinence de la généralisation de l'évaluation économique, l'affirmation de la légitimité des politiques publiques de biodiversité... Le projet de loi est clairement en décalage par rapport aux enjeux identifiés par cet exercice de prospective.

Sans doute, comme NSS l'a analysé dans son numéro spécial consacré à la conférence sur le climat qui se tiendra en décembre 2015 à Paris ${ }^{4}$, les résultats de cette conférence seront-ils plus décisifs en matière de transition écologique que cette loi de reconquête sans grandes ambitions ni moyens financiers.

Catherine Aubertin

\footnotetext{
4 Aubertin, C., Damian, M., Magny, M., Millier, C., Theys, J., Treyer, T. (Eds), 2015. Les enjeux de la conférence de Paris. Penser autrement la question climatique. Natures Sciences Sociétés, 23 (suppl. juin), http://www.nss-journal.org/articles/nss/abs/2015/ $02 /$ contents/contents.html.
} 